\title{
Aprender a gestionar emocionalmente la motivación de logro en Educación Primaria. Propuesta de intervención
}

José Santiago Álvarez Muñoz - Universidad de Murcia Mํán Aeles Hernández Prados - Universidad de Murcia

Noelia Carbonell Bernal - Universidad Internacional de La Rioja

Cristina Herrero Castellote - Universidad Internacional de La Rioja
D) $0000-0002-9740-6175$

$0000-0002-3617-215 \mathrm{X}$

(iD) $0000-0001-8113-6601$

(i) $0000-0002-4585-9957$

Recepción: 03.10.2021 | Aceptado: 19.102021

Correspondencia a través de ORCID: Máñngeles Hernández Prados

D 0000-0002-3617-215X

Citar: Álvarez Muñoz, JS, Hernández Prados, MA, Carbonell Bernal, N y Herrero Castellote, C (2021. Aprender a gestionar emocionalmente la motivación de logro en Educación Primaria. Propuesta de intervención. REIDOCREA, 10(40), 1-14.

Resumen: Educar en la motivación de logro es una premisa básica en la tarea del educador, y aprender a gestionarla emocionalmente una competencia a adquirir por los educandos para prevenir efectos colaterales perversos como frustración, desajustes en las expectativas, autoestima, sobrelevación del perfeccionismo y autoexigecia, etc. Constatado que los esfuerzos se centran más en la primera tarea, y no tanto en la segunda, se estima oportuno el diseño de un programa de intervención para promover el aprendizaje de la gestión emocional de la motivación de logro en los estudiantes de tercer ciclo de primaria. Proyecto compuesto por 25 actividades organizadas en 8 módulos en el que trabajan el autoestima, el autoconcepto y la gestión y control emocional desde una metodología de trabajo basada en el aprendizaje cooperativo-colaborativo, el uso de técnicas artísticas y la gamificación como vehículo metodológico para practicar lo emocional. El impacto de este tipo de intervención en el clima de aula, rendimiento académico, relaciones entre iguales, salud emocional, ha sido puesto de manifiesto en la bibliografía precedente, lo que permite concluir que resulta fundamental para el desarrollo personal de los estudiantes y para la mejora de la convivencia escolar.

Palabra clave: Educación Primaria

Learn to emotionally manage the motivation of achievement in Primary Education. Intervention proposal

\begin{abstract}
Educating achievement motivation is a basic premise in the task of the educator, and learning to manage it emotionally is a skill to be acquired by students to prevent perverse collateral effects such as frustration, mismatches in expectations, self-esteem, over-emphasis on perfectionism and self-demanding, etc. Given that efforts are focused more on the first task, and less on the second, it is considered appropriate to design an intervention programmed to promote the learning of emotional management of achievement motivation in third cycle primary school students. The project consists of 25 activities organized into 8 modules in which they work on self-esteem, self-concept and emotional management and control using a work methodology based on cooperative-collaborative learning, the use of artistic techniques and gamification as a methodological vehicle for practicing emotional skills. The impact of this type of intervention on classroom climate, academic performance, peer relations and emotional health has been highlighted in the previous bibliography, which leads to the conclusion that it is fundamental for the personal development of students and for the improvement of school coexistence.
\end{abstract}

Keyword: Primary Education

\section{Introducción}

La preocupación por la motivación escolar ha sido objeto de debates durante décadas. En los últimos años, se ha constatado que la mayoría de los alumnos presentan bajos niveles de motivación, lo que repercute negativamente en sus niveles de rendimiento académico, satisfacción social, clima escolar, relaciones interpersonales y colaborativas, autonomía, autosuficiencia, autoconcepto, convivencia escolar, entre otros aspectos (López-González y Oriol, 2016; Manzano y Valero-Valenzuela, 2019). 
Si bien los estudios se han centrado principalmente en los niveles de secundaria, de forma reciente se ha observado que la desmotivación de los alumnos hacia el aprendizaje escolar se inicia más tempranamente, aumentando el riesgo de fracaso escolar en los últimos años de la etapa de primaria. Al respecto, deben incrementarse los esfuerzos educativos por mantener activa en Secundaria los niveles de motivación que presentan estudiantes de Primaria en los últimos años (Manzano-Sánchez, 2021).

Se trata de intervenir preventivamente para evitar que la desmotivación se adueñe de los estudiantes de secundaria convirtiéndose en un satus quo. Para ello resulta esencial indagar en las posibles causas de la desmotivación. Ahora bien, los bajos niveles de motivación que presentan los estudiantes en la actualidad no encuentran explicación en una única causa. En contraposición, se trata de un problema complejo, multidimensional y poliédrico que dificulta enormemente cualquier intento de delimitación, identificación, análisis y tratamiento. Más allá de los aspectos curriculares y disciplinares (horas de clase, metodología, contenidos, criterios de evaluación, recursos didácticos, etc.) que indicen en la satisfacción que muestra el alumnado hacia el aprendizaje, también resulta importante la propia figura del docente, es decir, las capacidades, habilidades, interés y tacto que presenta en la relación con el otro (Baños et al, 2017).De ahí que ante esta problemática el profesorado no puede mirar hacia otro lado, por el contrario debe asumir la responsabilidad propia y trabajar colaborativamente con las familias para conseguir que los estudiantes despierten la motivación hacia el aprendizaje escolar.

Sin embargo, según los resultados obtenidos por Cáceres et al (2021) se observa, de forma generalizada, una mayor tendencia docente a la dejadez y desplazamiento de la responsabilidad de motivar a los estudiantes hacia la familia, por lo que se hace necesario generar estrategias para promover el compromiso docente continuado en esta función, entendiendo la motivación más allá de un momento específico del aula. Todo ello pone de relieve deficiencias formativas en los docentes para poder "generar contextos socioemocionales positivos y programas de desarrollo emocional del alumnado, lo cual beneficiará su rendimiento académico" (López-González y Oriol, 2016, p.130). Desde la pedagogía no podemos obviar esta demanda, y además de contribuir a proporcionar las competencias cognitivas y destrezas mediante encuentros formativos, resulta de gran utilidad diseñar propuestas para que éstos puedan desarrollar en sus aulas.

En definitiva, La queja constante de docentes y familias por la falta de motivación hacia el aprendizaje que presentan los estudiantes, es algo común que impide realizar un buen trabajo a los maestros. Una dificultad que plantea el reto educativo de lograr despertar el interés por la materia y las ganas de aprender, fomentar la motivación de logro, el orgullo por el trabajo bien hecho, lo emocionante de adquirir nuevos conocimientos, lo que engendró el siguiente problema de investigación ¿cómo conseguir que muestren disposición y motivación por aprender?

Haciéndonos eco de la recomendación de Manzano-Sánchez (2021) de la necesidad de realizar intervenciones que potencien la motivación, responsabilidad, necesidades psicológicas básicas y clima de aula en las últimas etapas de Primaria con el fin de favorecer el paso a Secundaria y mediar en las conductas antisociales y la violencia percibida, se ha diseñado la propuesta pedagógica que se expone en este trabajo. Aunque desafortunadamente, el Real Decreto 126/2014, de 28 de febrero para el currículo de Educación Primaria, y la normativa autonómica no contemplan directamente la educación emocional, si lo hace indirectamente, en la asignatura de valores sociales y cívicos, en los bloques: "Identidad y la dignidad de la persona", "Convivencia y valores sociales" y "Comprensión y respeto en las relaciones 
interpersonales". En cualquier caso, emociones y motivación, son aspectos de la persona que inciden en cualquier ámbito en el que esta se desarrolle, y se vincula con los objetivos a, b, c y d contemplados en el art.4 del Real Decreto 126/2014. Así mismo, en el art.5 sobre las competencias del currículo, se relacionan con el ámbito emocional y por consiguiente con la motivación, el aprender a aprender, las competencias sociales y cívicas, sentido de iniciativa y espíritu emprendedor, conciencia y expresiones culturales. De este modo queda justificado normativamente, la pertinencia de este tipo de intervención didáctica en los centros educativos. Antes de pasar a detallar todos los elementos que componen el programa de intervención, facilitando de ese modo su aplicabilidad en el aula, se expone a continuación las bases teóricas que lo sustentan.

\section{Fundamentos teóricos de la propuesta de intervención}

En los estudios sobre la motivación de la conducta humana, han tenido gran trascendencia la diferenciación entre dos tipos de motivación, intrínseca y extrínseca. La motivación intrínseca es aquella en la que el propio desarrollo de la actividad en sí misma constituye el objetivo y al tiempo la gratificación, provocando con su logro sensaciones de competencia y autorrealización. Se refiere a aquellos factores que provienen del interior de la persona y que son ajenos al contexto y a situaciones externas de la misma, que activan la conducta humana, y según Chóliz (2004) proporciona "sentimientos de competencia y autodeterminación" (p.38), mientras que la extrínseca responde a factores externos como premios o castigos. Aunque ambas se complementan, la Inteligencia Emocional y la motivación intrínseca, mientras que la correlación es negativa con la desmotivación y la regulación externa de la motivación (Fierro, Almagro y Sáenz-López, 2019). De igual modo, variables de inteligencia intrapersonal e interpersonal mostraron una correlación positiva con la motivación intrínseca (Vaquero et al., 2020). El interés por aprender está estrechamente ligado a la autoestima, por lo que en la medida en que se desarrolle la autoestima, también se incrementará su motivación y el deseo de aprender (Paredes y Moya, 2019). Si el docente logra despertar el interés por el conocimiento en sí mismo, se conseguirá la motivación más duradera y eficaz. El objetivo es por consiguiente que los alumnos disfruten y se impliquen espontáneamente. Para ello, deben sentirse capaces, autónomos y competentes.

La motivación es un constructo esencial en el aprendizaje y se relaciona también con otros aspectos como la autorrealización, situada por Maslow en la cúspide de la pirámide. Para cubrirla, se busca el desarrollo personal, de modo que cada individuo alcance el máximo potencial y el mayor desarrollo de sus capacidades, es decir, el logro de la excelencia. Al respecto, la teoría de expectativa-valor propuesta por Atkinson (1964), plantea tres motores conductuales en función de los cuales la persona estará más o menos motivada; lo que produce satisfacción o frustracióndesaliento: el motivo, expectativa-incentivo y las posibilidades de éxito. La búsqueda del logro se producirá en la confrontación de las tendencias opuestas al éxito y a la evitación del fracaso (Huertas, 1997). Para McClelland (1953) existen tres necesidades básicas que inciden en la motivación: la necesidad de afiliación, la de poder y la de logro, siendo esta última, el deseo del éxito y la excelencia en lo que uno hace, anhelo por sobresalir de los estándares.

Por otra parte, Rotter (1966) en su teoría del aprendizaje social desarrollo el constructo de locus de control como las creencias del individuo sobre las causas de sus acciones influyen en su actitud y conducta, para alcanzar una meta fijada. Existen dos tipos de locus de control, el interno se refiere a aquellas personas que se creen capaces de cambiar e influir en su entorno y externo, mostrando una postura activa; y el externo es 
propio de aquellos que piensan que su éxito depende de factores externos, comportándose pasivamente ante el entorno. Todas estas atribuciones de causalidad tienen efectos en el pensamiento, las emociones, el comportamiento, la autoestima y la motivación (Heider citado en Bisquerra, 2000). Combinando las teorías de Rotter, Heider y sus propias investigaciones, Weiner (1986) desarrolló su teoría atribucional en la que vinculaba las implicaciones emocionales y motivacionales con el éxito o el fracaso académico. "Las atribuciones causales efectuadas por un individuo condicionan sus expectativas y sus sentimientos, y ambos, expectativas y emociones, condicionan la acción" (Vázquez y Manassero, 1989, p. 230).

En lo que respecta a las emociones, el cerebro humano diferente al resto de especies, pues tiene la neocorteza prefrontal que permite tomar decisiones voluntarias y conscientes, y se encuentra influida por la emoción (Casafont, 2012). Las emociones son el motor que todos llevan dentro, y según Mora (2012) nos permite alejarnos de lo nocivo o acercarnos a lo placentero, motivan a conseguir algo o a evitarlo; la conducta se nos presenta flexible, polivalente, opcional y adaptativa; alertan a la persona; Despiertan y mantienen la curiosidad y el interés por el descubrimiento de lo nuevo; son una forma de comunicación; ayudan a la memoria a almacenar y evocar recuerdos; y influye en los razonamientos a considerar en la toma de decisiones. Por todo ello, se concibe como una modalidad de la inteligencia, definida la capacidad de conocer las emociones propias, manejarlas, reconocer las de los demás, motivarse y establecer relaciones positivas con los demás (Goleman, 1995). Dicho de otro modo, como:

"la habilidad para percibir, valorar y expresar emociones con exactitud, la habilidad para acceder $\mathrm{y} / \mathrm{o}$ generar sentimientos que faciliten el pensamiento; la habilidad para comprender emociones y el conocimiento emocional y la habilidad para regular las emociones proviniendo de un crecimiento emocional e intelectual" (Mayer y Salovey, 1997, p.4).

A pesar de los efectos positivos de la inteligencia emocional como la disminución de niveles de estrés, de conflictos y comportamientos de riesgo, mejora la salud mental y el equilibrio emocional, la resiliencia, la tolerancia a la frustración, el aprendizaje y por consiguiente, el rendimiento académico (Cervantes et al. 2018; Extremera y Fernández-Berrocal, 2001; Ibarrola, 2014; Mora, 2012), en los currículums escolares se prioriza los conocimientos académicos, relegando la educación emocional al ámbito privado. Si se habla de motivación de logro y emociones, no es posible obviar la influencia de las expectativas del maestro, ensalzadas en los trabajos de Merton (1948) sobre la profecía autocumplida y los de Rosenthal y Jacobson (1968) sobre el efecto Pigmalion, o sus efectos en el autoconcepto de los estudiantes. Existe una relación bidireccional entre motivación y emoción, es decir, las emociones generan motivos que llevan a la acción y la conducta motivada genera reacciones emocionales (Estrada, 2018), de ahí que, el maestro tiene que conocer el estado emocional de sus estudiantes y emplear ese conocimiento para las prácticas docentes (Lindqvist et al., 2017).

La motivación determina el grado de implicación en la tarea, la curiosidad, la atención, el esfuerzo, etc. y todo ello da lugar a unos resultados académicos determinados. Si se logra desarrollar en los niños una motivación de logro, se les estará capacitando para una trayectoria académica sobresaliente, pero también para lograr los objetivos profesionales y personales que se marquen a lo largo de toda su vida. Establecer programas de intervención donde el maestro se centre en motivar al alumno hacia sus propios logros académicos, conociendo a sus estudiantes, creando un clima positivo para mejorar su rendimiento, es una importante tarea docente, según el estudio de Veas, Castejón, Gilar y Miñano (2017). 
A continuación de forma sintetizada, se muestran, en la tabla siguiente, las principales teorías en las que se sustenta esta investigación.

TABLA 1. TEORÍAS MOTIVACIONALES Y DE INTELIGENCIA EMOCIONAL

\begin{tabular}{l} 
TEORIAA Y AUTOR \\
\hline LA JERARQUIAA DE \\
NECESIDADES DE \\
MASLOW \\
LA TEORÍA DE \\
EXPECTATIVA-VALOR \\
DE ATKINSON \\
LA TEORÍA DE LAS TRES \\
MOTIVACIONES DE \\
MCCLELLAND
\end{tabular}

LA TEORÍA DEL APRENDIZAJE SOCIAL DE ROTTER Y EL LOCUS DE CONTROL

EN LA TEORÍA ATRIBUCIONAL DE WEINER

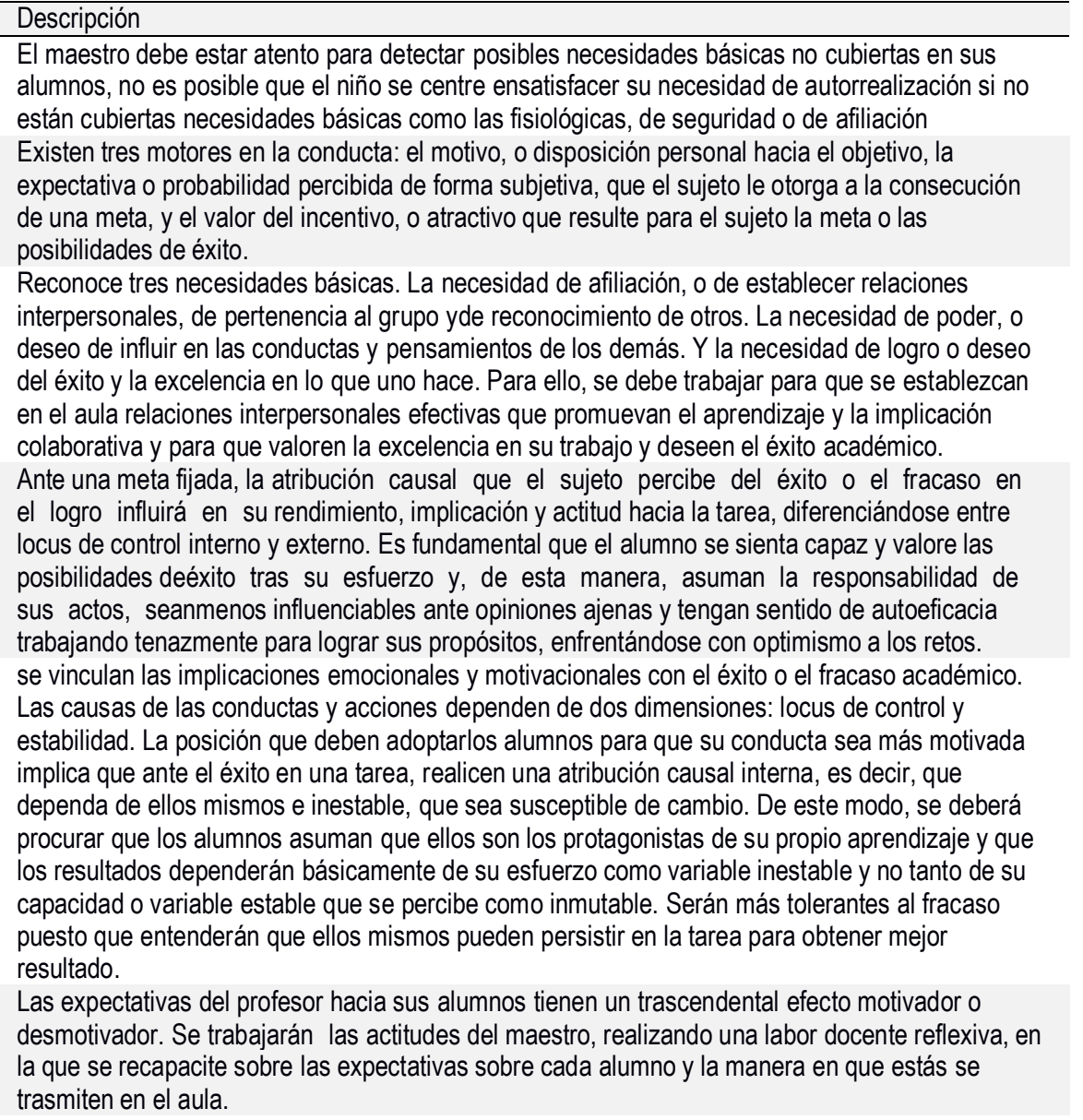
Descripción alumnos, no es posible que el niño se centre ensatisfacer su necesidad de autorrealización si no expectativa o probabilidad percibida de forma subjetiva, que el sujeto le otorga a la consecución de una meta, y el valor del incentivo, 0 atractivo que resulte para el sujeto la meta o las ilidades de éxito. interpersonales, de pertenencia al grupo yde reconocimiento de otros. La necesidad de poder, 0 deseo de influir en las conductas y pensamientos de los demás. Y la necesidad de logro o deseo del éxito y la excelencia en lo que uno hace. Para ello, se debe trabajar para que se establezcan colaborativa y para que valoren la excelencia en su trabajo y deseen el éxito académico. el logro influirá en su rendimiento, implicación y actitud hacia la tarea, diferenciándose entre locus de control interno y externo. Es fundamental que el alumno se sienta capaz y valore las posibilidades deexito tras su esfuerzo y, de esta manera, asuman la responsabilidad de se vinculan las implicaciones emocionales y motivacionales con el éxito o el fracaso académico. Las causas de las conductas y acciones dependen de dos dimensiones: locus de control y estabilidad. La posición que deben adoptarlos alumnos para que su conducta sea más motivada dependa de ellos mismos e inestable, que sea susceptible de cambio. De este modo, se deberá capacidad o variable estable que se percibe como inmutable. Serán más tolerantes al fracaso puesto que entenderán que ellos mismos pueden persistir en la tarea para obtener mejor

DE ROSENTHAL Y JACOBSON

\section{EL EFECTO PIGMALION}

la que se recapacite sobre las expectativas sobre cada alumno y la manera en que estás se trasmiten en el aula.

\section{Objetivos}

Este trabajo tiene como finalidad diseñar una secuencia didáctica para que el alumnado de tercer ciclo de primaria aprenda las habilidades necesarias que le permitan gestionar emocionalmente la motivación de logro. Más específicamente, en la delimitación de los objetivos específicos quedan identificadas dichas habilidades.

- OE1.Crear pautas de comunicación asertiva entre los alumnos y con el maestro.

- OE2. Fomentar la autoestima, el autoconcepto y la autonomía.

- OE3. Establecer pautas de actuación y pensamiento que fomenten el desarrollo de lamotivación hacia el logro de metas personales.

- OE4. Valorar los logros individuales, propios y de los demás.

- OE5. Reconducir la conciencia deseada hacia el locus de control.

- OE6. Identificar las expectativas personales y asignarles un valor proporcional y adecuado.

- OE7. Descubrir las emociones propias y ajenas y ordenarlas jerárquicamente, relacionandolas emociones con sus causas.

- OE8. Afianzar las potencialidades de la persona. 


\section{Metodología}

Todo el entramado de actividades queda sustentado bajo un marco metodológico que se caracteriza por ser: global, no se entiendo por áreas diferenciadas, sino que comprende aprendizajes integrales; activo, en todo momento la participación de los alumnos resulta vital para el desarrollo de la intervención; y, por último, dinámica, se ofrecen diferentes tipos de actividades, agrupamientos y recursos que incrementan el atractivo. De forma más específica, en lo que respecta a lo emocional, se sitúa un aprendizaje colaborativo y cooperativo que pone en alza el valor de las vivencias y experiencias donde las relaciones sociales son una constante, siendo el proceso de la socialización la llave que da acceso a abordar el trabajo emocional.

Por otro lado, en lo que respecta a los agrupamientos, se utilizan las siguientes modalidades: pequeños grupos, a partir del cual abrir paso al debate y confrontación de ideas para dar paso al aprendizaje; las parejas, tareas realizadas en común para el refuerzo y consolidación de los contenidos; por último, individualmente, dejando momentos para la reflexión y autoconocimiento, dotando de un tiempo para el "yo" que posibilite asumir lo aprendido.

Finalmente, concretizando el método, sustentado en la base metodológica y los agrupamientos ideados, se establece un método de trabajo en consonancia con los objetivos y contenidos relativos a la educación emocional, subrayando los siguientes componentes:

- Aprendizaje dialógico: subrayando la importancia de la expresión y comprensión oral como vehículo comunicativo de las emociones y sentimientos, representándose como llave paso para una multitud de aprendizajes.

- La reflexión: Con el propósito de poder canalizar los aprendizajes resulta más que necesario proceder a una reflexión tras la realización de una tarea. De esta forma, se podrá asentar todos los conocimientos a los que el alumnado ha estado en contacto durante la actividad principal. Se puede presentar de forma individual o grupal.

- Talleres: Toda acción desarrollada se da bajo carácter práctico que otorga un plus de funcionalidad y significatividad a los contenidos instruidos.

- Creatividad: La creación resulta un componente crucial visible desde la imaginación y el arte como medios para su ejercicio.

- Lúdico: La diversión asociada al aprendizaje lo hace más ameno y enriquecedor, además invita a una actitud más positiva y motivadora frente a las actividades.

\section{Actividades y temporalización}

Atendiendo a los objetivos específicos y en base a tres teorías explicativas del ámbito de la psicología educativa se exponen y organizan un total de 25 actividades (Tabla 1 ). En primer lugar, la teoría de Weiner que relaciona el resultado de la actividad y su posterior reflexión con el aprendizaje de la emoción, mejorando el control y gestión de esta ante la consecución o no del logro. En segundo lugar, el modelo de inteligencia emocional de Mayer y Salovey destaca las habilidades de percepción, asimilación y comprensión emocional como requerimientos previos para poder acceder a la gestión emocional. Por último, las expectativas del profesor en la autoestima y la motivación, como eje de influencia en el desarrollo del autoconcepto de los alumnos. 
Tabla 1. Actividades, objetivos específicos y descripción según módulos temáticos

\begin{tabular}{|c|c|c|}
\hline \multicolumn{3}{|c|}{ Modulo centrado en la jerarquía de las necesidades } \\
\hline $\begin{array}{l}\text { Actividad } 1 . \\
\text { Las necesidades } \\
\text { básicas }\end{array}$ & OE6, E01 & $\begin{array}{l}\text { Con la técnica de folio giratorio en grupos irán escribiendo las necesidades humanas y, } \\
\text { además, ordenando de acuerdo a su mayor o menor importancia. Posteriormente, } \\
\text { debatirán y justificarán sus decisiones. }\end{array}$ \\
\hline $\begin{array}{l}\text { Actividad } 2 . \\
\text { ¿Qué } \\
\text { necesidades? }\end{array}$ & OE6, OE1 & $\begin{array}{l}\text { Se anotarán las necesidades en unas fichas para ponerlas boca abajo sobre una mesa. Uno a } \\
\text { uno irá cogiendo una para representarla con mímica al resto de compañeros, posteriormente, } \\
\text { hablarán sobre las consecuencias de su satisfacción o no. }\end{array}$ \\
\hline $\begin{array}{l}\text { Actividad } 3 . \\
\text { Collage de } \\
\text { necesidades }\end{array}$ & OE6, OE1 & $\begin{array}{l}\text { Buscarán fotos de revistas donde aparezcan personas que, según su percepción, tengan una } \\
\text { necesidad sin cubrir. Lo pegarán en una cartulina explicando la necesidad para, con todas } \\
\text { juntas, conformar un collage. Finalmente, se comentará en gran grupo. }\end{array}$ \\
\hline \multicolumn{3}{|c|}{ Modulo centrado en la expectativa valor } \\
\hline $\begin{array}{l}\text { Actividad } 4 . \\
\text { Mi objetivo } \\
\text { personal }\end{array}$ & OE4, OE6 & $\begin{array}{l}\text { Cada alumno pensará en cómo serán ellos en el futuro. Acto seguido, escribirán una carta } \\
\text { a su "yo" del futuro aportándole recomendaciones y avisos para lograr las metas que } \\
\text { quieren. Luego se leerá en público a fin de enriquecer su aportación con el resto de los } \\
\text { compañeros. }\end{array}$ \\
\hline $\begin{array}{l}\text { Actividad } 5 \text {. } \\
\text { No todas las } \\
\text { metas son igual de } \\
\text { fáciles }\end{array}$ & OE4, OE6 & $\begin{array}{l}\text { Habrá un listado de metas y, por grupos, deben ordenarlas por dificultad. Después de ello, en } \\
\text { grupo debatirán sobre su elección. }\end{array}$ \\
\hline
\end{tabular}

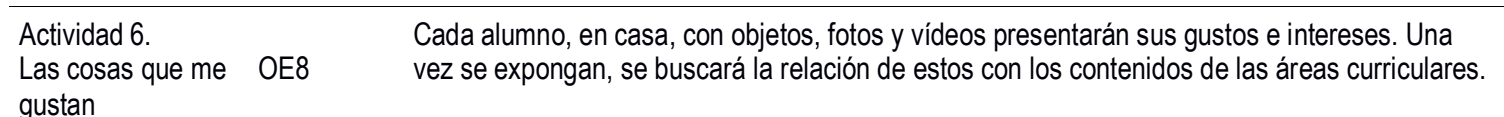

\section{Modulo centrado en las necesidades de filiación}

Actividad 7.

El anuncio
En parejas han redactar un anuncio para intentar convencer a los companeros de la OE1, OE2, compra de un producto determinado, en este caso, su otro compañero. Se han de detallar OE4 sus cualidades, éxitos o potencialidades por medio de la creación de un video corto promocional.

\begin{tabular}{lll}
\hline $\begin{array}{l}\text { Actividad } 8 . \\
\begin{array}{l}\text { El logo de la } \\
\text { clase }\end{array}\end{array}$ & OE4 & $\begin{array}{l}\text { Se ha de idear un logotipo representativo de la clase por grupos. Luego se dará paso a } \\
\text { una defensa para decidir, por medio de una votación, el logo final que represente a la } \\
\text { clase. }\end{array}$
\end{tabular}

Actividad 9. La clase se divide en 3 grupos de 7. Irán dispuestos en fila, todos con los ojos tapados a El tren loco OE1 excepción del último pues es el que ha de guiarlos por un camino concreto. Cuando lo hagan los tres grupos, se comentarán en gran grupo sobre las técnicas o estrategias utilizadas.

Actividad 10. Diferentes pero iguales

Se harán grupos y estos deben buscar diez cosas en común más allá de lo físico, además

OE4, OE2 de buscar un nombre a partir de esa información. Finalmente se aportará en común para poder dotar de sugerencias.

Modulo centrado en las necesidades de logro

\begin{tabular}{|c|c|c|}
\hline $\begin{array}{l}\text { Actividad } 11 . \\
\text { Consigo mis } \\
\text { metas }\end{array}$ & $\begin{array}{l}\text { OE3, OE5, } \\
\text { OE6 }\end{array}$ & $\begin{array}{l}\text { Harán una tormenta de ideas sobre sus metas de vida, organizando después en base a } \\
\text { cuatro categorías: estudios, carácter, familia y amigos. Posteriormente, elegirán dos para } \\
\text { articular una estrategia para su consecución. }\end{array}$ \\
\hline $\begin{array}{l}\text { Actividad } 12 . \\
\text { Lo que quiero } \\
\text { lograr }\end{array}$ & $\begin{array}{l}\text { OE3, OE5, } \\
\text { OE6 }\end{array}$ & $\begin{array}{l}\text { Se realiza una tormenta de ideas sobre aspectos a mejorar en el ámbito académico, } \\
\text { debiendo elegir tres del conjunto. Acto seguido, deben pensar estrategias para su mejora, } \\
\text { determinando plazos y objetivos para su consecución. }\end{array}$ \\
\hline $\begin{array}{l}\text { Actividad } 13 . \\
\text { Soy el mejor }\end{array}$ & $\begin{array}{l}\text { OE1,OE3, } \\
\text { OE5, OE6 }\end{array}$ & $\begin{array}{l}\text { Los alumnos deben compartir algún logro personal del que se sientan orgullosos, } \\
\text { explicando los pasos para su consecución. Tras cada relato, el resto de compañeros } \\
\text { comentarán aquello que más han valorado. }\end{array}$ \\
\hline $\begin{array}{l}\text { Actividad } 14 . \\
\text { Todos somos } \\
\text { capaces mejorar }\end{array}$ & OE4 & $\begin{array}{l}\text { Cada alumno ha de seleccionar un personaje familiar o no, escribiendo una característica } \\
\text { personal que admiren. Posteriormente, debatirán si dichos personajes son dignos de } \\
\text { admiración haciendo hincapié en el esfuerzo, la tenacidad, la superación y el valor } \\
\text { educativo de los errores. }\end{array}$ \\
\hline \multicolumn{3}{|c|}{ Modulo centrado en el control interno } \\
\hline $\begin{array}{l}\text { Actividad } 15 . \\
\text { Mi medalla }\end{array}$ & OE1 & $\begin{array}{l}\text { Personalmente, pensarán un logro personal del que se sientan orgullosos, dándole una } \\
\text { medalla en honor por ese triunfo, la cual harán y se pondrán ellos mismos. Luego, en gran } \\
\text { grupo, se explicará y felicitará en común por ello. }\end{array}$ \\
\hline
\end{tabular}




\begin{tabular}{|c|c|c|}
\hline $\begin{array}{l}\text { Actividad } 16 . \\
\text { Tú tienes la llave }\end{array}$ & $\begin{array}{l}\text { OE1, OE3, } \\
\text { OE4. }\end{array}$ & $\begin{array}{l}\text { Se le pondrá un listado de metas, de gran atractivo y, en grupo, han de ordenar de acuerdo a } \\
\text { su accesibilidad. Finalmente, se debatirá en grupo a fin de acotar que la dificultad es relativa. }\end{array}$ \\
\hline \multicolumn{3}{|c|}{ Modulo centrado en la atribución causal } \\
\hline $\begin{array}{l}\text { Actividad } 17 . \\
\text { ¿Cuál es la } \\
\text { causa? }\end{array}$ & OE5 & $\begin{array}{l}\text { Se proyectará un listado de proposiciones por parejas, cuya elección identifica un locus de } \\
\text { control interno o externo. Deberán responderlas individualmente en un folio seleccionando cuá } \\
\text { es en su opinión la causa correcta. Se compartirán con la clase las respuestas y aquellas que } \\
\text { sean discrepantes se argumentarán, reflexionando sobre el tipo de locus de control tiene cada } \\
\text { uno y cuál sería el más adecuado en cada circunstancia. }\end{array}$ \\
\hline $\begin{array}{l}\text { Actividad 18. Las } \\
\text { noticias }\end{array}$ & OE5 & $\begin{array}{l}\text { Cada niño/a llevará una noticia procedente de una fuente fiable para que se lo expliquen a } \\
\text { sus compañeros indicando cuál es la causa del acontecimiento y quién es el responsable } \\
\text { de que ocurriera. Se debatirán las distintas posiciones y el docente guiará el debate } \\
\text { para que se hagan las atribuciones causales adecuadas. }\end{array}$ \\
\hline \multicolumn{3}{|c|}{ Modulo centrado en la percepción, asimilación y gestión emocional } \\
\hline $\begin{array}{l}\text { Actividad } 19 . \\
\text { Mis emociones }\end{array}$ & OE1, OE7 & $\begin{array}{l}\text { Cada alumno escribirá acerca de una emoción experimentada en una vivencia o sueño. } \\
\text { Aquellos alumnos que lo deseen, expondrán su vivencia y emociones, el resto } \\
\text { escucharán sin hacer juicios. }\end{array}$ \\
\hline $\begin{array}{l}\text { Actividad 20. Qué } \\
\text { siento }\end{array}$ & OE1, OE7 & $\begin{array}{l}\text { En grupos comentarán como se sienten ante diferentes situaciones desafiantes que } \\
\text { pueden ocurrir en el día a día. Se pondrán en común las diferentes respuestas y se } \\
\text { establecerá un debate sobre aquellas emociones que no coincidan entre los grupos, y los } \\
\text { miembros de cada uno explicarán el porqué de su elección. }\end{array}$ \\
\hline $\begin{array}{l}\text { Actividad } 21 . \\
\text { Me saca de mis } \\
\text { casillas }\end{array}$ & OE1, OE7 & $\begin{array}{l}\text { El docente mostrará las siguientes frases: "Recuerdo una situación que me saca de mis } \\
\text { casillas", "Recuerdo una situación que me provoca alegría", "Recuerdo una situación que } \\
\text { me produce miedo", "Recuerdo una situación que me deja cabizbajo/a" y deberán } \\
\text { responder por escrito a las siguientes preguntas: ¿Quién crea la situación?, } \\
\text { ¿Cuándo y dónde sucede?, ¿Qué hago yo? y ¿Cómo respondo?, ¿Qué siento entonces? } \\
\text { Y yo hago...Finalmente, se expondrán las respuestas y se debatirá sobre cuál es la } \\
\text { reacción más adecuada a cada situación }\end{array}$ \\
\hline \multicolumn{3}{|c|}{ Modulo centrado en el fomento de la autoestima } \\
\hline $\begin{array}{l}\text { Actividad } 22 . \\
\text { Así soy }\end{array}$ & OE2, OE4. & $\begin{array}{l}\text { Cada niño dibujará en lo alto de un papel las palabras "así soy yo". Debajo deberán } \\
\text { dibujar todo lo que les defina. Puede ser un retrato o palabras sueltas, adjetivos, dibujos, } \\
\text { símbolos... Después saldrán a la pizarra y explicarán su dibujo al resto de la clase y el } \\
\text { porqué de cada elemento. }\end{array}$ \\
\hline $\begin{array}{l}\text { Actividad } 23 . \\
\text { Fotografía de mi } \\
\text { momento }\end{array}$ & OE1, OE7 & $\begin{array}{l}\text { Los alumnos tendrán que traer de casa una fotografía de un momento elegido, } \\
\text { especialmente emotivo para ellos. Deberán mostrar y comentar por qué la han elegido y } \\
\text { cómo se sintieron mientras vivían aquella situación. }\end{array}$ \\
\hline $\begin{array}{l}\text { Actividad } 24 . \\
\text { Cómo me veo y } \\
\text { cómo me ven }\end{array}$ & OE2, OE4 & $\begin{array}{l}\text { Cada alumno escribirá en un papel todos sus puntos más fuertes y dejará el folio } \\
\text { bocabajo. Después se colocarán un folio pegado a la espalda y se desplazarán por la } \\
\text { clase mientras los compañeros escriben en la espalda de unos u otros, los aspectos que } \\
\text { valoren positivamente de cada compañero. A continuación, compararán lo que ellos han } \\
\text { escrito con lo que han escrito sobre ellos sus compañeros. }\end{array}$ \\
\hline $\begin{array}{l}\text { Actividad } 25 . \\
\text { El árbol }\end{array}$ & $\begin{array}{l}\text { OE4, OE6, } \\
\text { OE3. }\end{array}$ & $\begin{array}{l}\text { Los alumnos deberán construir un árbol como deseen poniendo una foto suya en el tronco } \\
\text { y en las raíces deberán poner cualidades o características personales positivas. Además, } \\
\text { harán hojas que colgarán de sus ramas donde en cada una indicar un logro personal. Los } \\
\text { árboles se expondrán en el aula. }\end{array}$ \\
\hline
\end{tabular}

Esta intervención ha sido idea para ser desarrollada durante un curso académico, concretamente se prevé la realización de 3 o 4 actividades por mes, desde octubre a mayo, dejando los periodos de septiembre para la preparación previa y el mes de junio para su evaluación (Anexo I).

\section{Procedimientos e instrumentos de evaluación}

Tomando la evaluación como la oportunidad de mejora, se propone una evaluación continua y formativa durante todo el proceso desarrollada mediante la observación sistemática y directa, lo que permitirá un conocimiento más completo del aprendizaje. No obstante, se contemplan diferentes momentos de evaluación. 
Una evaluación inicial o diagnóstica en la que los alumnos completarán el "Cuestionario de evaluación motivacional del proceso de aprendizaje" que nos permite obtener un Índice EMPA (Blasco et al, 2016). Se trata de una escala tipo liker, compuesto de 32 items y 5 opciones de respuesta que mide la motivación extrínseca, intrínseca y global, lo que permite una obtención de información muy útil para valorar las necesidades y poder seleccionar y ajustar la intervención al contexto específico del centro.

Una evaluación procesal de las sesiones, recursos y actividades, tomando las notas necesarias que se incorporarán al diario del profesor con la finalidad de identificar las fortalezas, debilidades y propuestas de cambio. El diario de campo permite disponer de la información precisa para elaborar un registro de observación de los alumnos (Anexo II).

Por otro lado, dada la decisiva influencia de la práctica docente en la motivación de los educandos, se plantea la realización de una autoevaluación mediante un cuestionario ad hoc (Anexo III), cuyo objetivo es la reflexión personal sobre la propia práctica, valorando aspectos cognitivos, procedimentales y emocionales. Cualquier metodología a implantar requiere una reflexión de la práctica docente y del aprendizaje estudiantil, aportando eficacia y mejora a la labor educativa.

Por ultimo, se debe llevar a cabo la evaluación final para contrastar los resultados obtenidos y el impacto generado, en el que se vuelve a aplicar el instrumento inicial, incorporando la valoración del proceso global. No se puede olvidar que lo que se pretende es una mejora en las emociones, autoestima, atribuciones y motivación y todo ello afectará a cualquier ámbito en el que se encuentre el niño. Para tener constancia de ello se aplica el mismo test antes y después de la intervención.

\section{A modo de conclusión}

El tema de la motivación ha adquirido un gran protagonismo en el ámbito laboral, pero en el ámbito escolar, su inclusión como elemento no cognitivo y emocional lo ha relegado en ocasiones, a ser un aspecto emocional entre otros muchos, perdiendo bastante de su especificidad. Además, las experiencias de intervención en educación emocional contribuyen entre sus logros a la mejora de la motivación como apunta Pérez-González (2012) en la revisión realizada, de modo que trabajando lo primero se consigue lo segundo, haciendo parecer innecesario la intervención en esta última. En contraposición, en el programa que hemos presentado otorgamos una mayor relevancia a lo motivacional situándolo en el epicentro de la intervención. Entendemos emoción-motivación como dos conceptos complementarios, ya que las "estrategias afectivomotivacionales se caracterizan por generar cogniciones y emociones en los estudiantes con respecto a las actividades de aprendizaje" (Dorado-Martínez et al, 2020, p.79).

La sistematicidad que define la organización de este proyecto, "visualizada como una posibilidad para producir conocimiento a partir de la intervención en la realidad" (Carvajal-Burbano, 2018, p.13), junto a la coherencia y congruencia que mantiene con la fundamentación teórica son dos principios que avalan el buen funcionamiento de los programas en educación, y más específicamente en lo emocional-motivación (Garaigordoibil, 2018). Se pone de manifiesto así la necesidad de una coherencia teórico - práctica en cuanto al diseño y puesta en práctica de intervenciones en el ámbito educativo, pues de cualquier otro modo, se corre el peligro de no cumplir los propósitos marcados o no adecuar la intervención a los destinatarios, tal y como se aprecia en los trabajos de Gutierrez-de Rozas y Carpintero (2021) y Torrijos et al 
(2018). Por lo cual, la revisión documental o bibliográfica como paso previo en el desarrollo de proyectos educativos no ha de ser menospreciado, sino que debe ser considerado como uno de los pasos claves para asegurar el éxito posterior.

En cuanto a la intervención, cabe remarcar dentro del engranaje metodológico la vital importancia que se le da al aprendizaje colaborativo y cooperativo. Resulta necesario que en el ámbito de lo emocional el colaborar, vivenciar, compartir y comunicar sean acciones constantes en el itinerario de actividades, los destinatarios han de dar voz a sus emociones más profundas de manera que estos también adquieran la capacidad y madurez de poder transmitirlas, aprendizaje que resulta más arduo de adquirir que la gestión o control emocional. Además, es característico de una etapa evolutiva significativa, la adolescencia, en la cual los menores se sienten retraídos para hacer testimonio de su vida personal, siendo prioritario la transmisión de habilidades, pautas y capacidades para poder hacerlo a nivel grupal (Vaquero-Solís et al, 2020).

La organización por módulos hace posible la determinación de las actividades en consonancia a los objetivos y contenidos planteados para el proyecto educativo. La delimitación de lo que se trabaja en un momento u otro queda clarificado ante este tipo de planificación del trabajo pues permite una focalización específica que resulta inviable cuando se desarrolla desde un tratamiento global. Además, al estar desarrollados según lo establecido en los objetivos específicos, existe una importante correspondencia entre este proceder de organización y el proceso de evaluación de manera que resulta sencillo comprobar el grado de adquisición de estos.

También cabe destacar la importancia que guarda el protagonismo juvenil en el desarrollo de las actividades pues los adolescentes son los artífices de todo lo que concurre. Se parte de la vivencia personal para poder dar pie al desarrollo posterior en el ámbito grupal de manera que el rol de los menores queda representado como variable de importante consideración (Baños et al, 2017). Partir del testimonio personal también posibilita dar un servicio de personalización de la enseñanza adecuándose a las necesidades de cada discente pues, sobre todo en el ámbito emocional, resulta prioritario articular una praxis que permite al docente ser conocedor de la realidad personal para poder intervenir desde una realidad comunitaria. Además, la mejora emocional a nivel individual revierte positivamente sobre el clima de clase y la convivencia entre los compañeros, estos adquieren herramientas para poder gestionar los conflictos y desarrollar relaciones basadas en la empatía y la comunicación (Manzano-Sánchez,2021).

Finalmente, este proyecto puede ser aplicado desde diferentes escenarios de escolaridad tanto a nivel presencial como virtual. Principalmente está enfocado a ser desarrollado a nivel grupal en el aula, pero se pueden diseñar actividades complementarias a poner en práctica con la familia. De esta forma, se posibilita en trabajo emocional desde otro agente social de influencia: la familia, con la cual también existen importantes lagunas para establecer vínculos emocionales, debilidad que se hace más patente en el periodo de la adolescencia. Aunque sea fácil de poder articular a otra realidad escolar no podemos obviar la importancia de hacer un análisis contextual inicial para poder ajustar a las nuevas demandas o necesidades que emanan desde este nuevo entorno de trabajo, modificando o incluyendo nuevos elementos que haga posible la personalización de este proyecto para adecuarse a la nueva realidad educativa.

\section{Referencias}


académico en estudiantes de Secundaria y Bachillerato: antecedentes, diseño, metodología y propuesta de análisis para un trabajo de investigación. Espiral. Cuadernos del Profesorado, 10(20), 40-50.

Bisquerra, R. (2000). Educación emocional y bienestar. Wolters Kluwer.

Cáceres, C., Muñoz, C., y Valenzuela, J. (2021). Responsabilidad personal docente y motivación escolar. Revista electrónica interuniversitaria de formación del profesorado, 24(1), 175-188.

Carvajal Burbano, A. (2018). Teoría y práctica de la sistematización de experiencias. Programa Editorial UNIVALLE.

Casafont, R. (2012). Viaje a tu cerebro emocional. Grupo Zeta.

Cervantes, D. I., Valadez, M. D., Valdés, A. A., y Tánori, J. (2018). Diferencias en autoeficacia académica, bienestar psicológico y motivación al logro en estudiantes universitarios con alto y bajo desempeño académico. Psicología desde el caribe, 35(1), 7-17.

Chóliz, M. (2004): Psicología de la Motivación: el proceso motivacional.

Dorado-Martínez, Á., Yandar, J. A., Garcez-Muñoz, Y., \& ObandoGuerrero, L. M. (2020). Programa de estrategias de aprendizaje para estudiantes de una institución educativa. Praxis \& Saber, 11(25), 75-95.

Estrada, L. (2018). Motivación y Emoción. Fundación Universitaria del Área Andina.

Fierro Suero, S., Almagro, B. J., ySáenz-López Buñuel, P. (2019). Necesidades psicológicas, motivación e inteligencia emocional en Educación Física. Revista Electrónica Interuniversitaria de Formación del Profesorado, 22(2).

Garaigordobil, M. (2018). La educación emocional en la infancia y la adolescencia. Participación Educativa, 5(8), 105-128.

Gardner, H. (2012): La educación de la mente y el conocimiento de las disciplinas. Paidós.

Goleman, D. (1995). Inteligencia emocional. Kairós.

Gutiérrez-de-Rozas, B. y Carpintero Molina, E. (2021). Análisis de la evaluación de programas de intervención en motivación en Educación Secundaria. Revista de Investigación Educativa, 39(2), 503-525.

Huertas, J. A. (1997) Motivación. Querer aprender: Aique.

Leal, F. (2018). Conducta motivada en la escuela: una revisión de motivación de logro desde la teoría de meta.: Claves Para El Asesoramiento Psicoeducativo. Temas En Psicología

Educacional. Contribuciones Para La Formación De Especialidad, Vol. 1. NOVEDUC.

Lindqvist H., Weurlander M., Wernerson A., Thornberg R. (2017) Resolving feelings of professional inadequacy: Student teachers' coping with distressful situations. Teaching and Teacher Education, 64, 270-279.

\section{Anexos}

\section{ANEXO I. Temporalización}

López-González, L., y Oriol, X. (2016). La relación entre competencia emocional, clima de aula y rendimiento académico en estudiantes de secundaria. Cultura y Educación, 28(1), 130156.

Manzano-Sánchez, D. (2021). Diferencias entre aspectos psicológicos en Educación Primaria y Educación Secundaria. Motivación, Necesidades psicológicas básicas, Responsabilidad, Clima de aula, Conductas antisociales y Violencia. Espiral. Cuadernos del Profesorado, 14(28), 9-18. https://doi.org/10.25115/ecp.v14i28.4198

Manzano-Sánchez, D. y Valero-Valenzuela, A. (2019). El Modelo de Responsabilidad Personal y Social (MRPS) en las diferentes materias de la Educación Primaria y su repercusión en la responsabilidad, autonomía, motivación, autoconcepto y clima social. Journal of Sport and Health Research, 11(3), 273-288.

McClelland, D. Atkinson,J.W., Clark, R.A.and Lowell, E.L. (1953). The achievement motive. Appleton-Century-Crofts.

Merton, R. (1948). The Self-Fulfilling Prophecy. The Antioch Review, 8(2), 193-210.

Pérez-González, J. C. (2012). Revisión del aprendizaje social y emocional en el mundo. En R. Bisquerra (Coord.), ¿Cómo educar las emociones? La inteligencia emocional en la infancia y la adolescencia (pp. 56-69). Esplugues de Llobregat.

Ramírez, J. M. G. (2016). La motivación de logro mejora el rendimiento académico. REIDOCREA, 5(1), 1-8.

Rosenthal, R. y Jacobson, L. (1968) Pygmalion en la escuela. Expectativas del maestro y desarrollo intelectual del alumno. Marova.

Rotter, J.B. (1996) Generalized expectancies for internal versus external control ofreinforcement. Psychological Monographis, $80(1), 1-28$.

Torrijos, P., Torrecilla, E. M., y Rodríguez, M. J. (2018). Experimental evaluation of emotional development programmes for teachers in Secondary Education. Anales de psicología, 34(1), 68-76.

Vaquero-Solís, M., Amado Alonso, D., Sánchez-Oliva, D., Sánchez-Miguel, P., y Iglesias- Gallego, D. (2020). Inteligencia emocional en la adolescencia: motivación y actividad física. Revista Internacional de Medicina y Ciencias de la Actividad Física y del Deporte, 20(77), 119-131.

Vázquez, A y Manassero, MA (1989). Teoría de la atribución y el rendimiento escolar. Educación y Cultura, 7, 225-241.

Veas, A., Castejón, J.-L., Miñano, P., y Gilar-Corbí, R. (2019). Actitudes en la adolescenciainicial y rendimiento académico: el rol mediacional del autoconcepto académico. Revista de Psicodidáctica, 24(1), 71-77.

Weiner, B. (1986). An attributional theory of motivation and emotion. Springer-Verlag. 


\begin{tabular}{|c|c|}
\hline Octubre & $\begin{array}{l}\text {-Semana 1a: Actividad } 1 \\
\text {-Semana 2a: Actividad } 9 \\
\text {-Semana 3a: Actividad - } 17 \text { - Primera Sesión } \\
\text {-Semana 4a: Actividad - } 17 \text { - Segunda Sesión }\end{array}$ \\
\hline & $\begin{array}{l}\text {-Semana 5a: Actividad } 2 \\
\text {-Semana 6a: Actividad } 10 \\
\text {-Semana 7a: Actividad - } 18 \text {-Primera Sesión } \\
\text {-Semana 8a: Actividad - 18- Segunda Sesión }\end{array}$ \\
\hline Dic & $\begin{array}{l}\text {-Semana 9a: : Actividad } 3 \\
\text {-Semana 10: Actividad } 11 \\
\text {-Semana 11: Actividad } 19 \\
\text {-Semana 12a: Actividad } 25\end{array}$ \\
\hline & $\begin{array}{l}\text {-Semana 13a: Actividad } 4 \text { - Primera Sesión } \\
\text {-Semana 14a: Actividad } 4 \text { - Segunda Sesión } \\
\text {-Semana 15a: Actividad } 12 \\
\text {-Semana 16: Actividad } 20\end{array}$ \\
\hline & $\begin{array}{l}\text {-Semana 17a: Actividad } 5 \\
\text {-Semana 18a: Actividad } 13 \text { - Primera Sesión } \\
\text {-Semana 19a: Actividad } 13 \text { Segunda Sesión } \\
\text {-Semana 20a: Actividad } 21\end{array}$ \\
\hline VIarzo & $\begin{array}{l}\text {-Semana 21a: Actividad 6 - Primera Sesión } \\
\text { - Semana 22a: Actividad 6- Segunda Sesión } \\
\text {-Semana 23a: Actividad 6- Tercera Sesión } \\
\text {-Semana 24a: Actividad } 14\end{array}$ \\
\hline ADIII & $\begin{array}{l}\text {-Semana 25a: Actividad } 7 \\
\text {-Semana 26a: Actividad } 15 \text { - Primera Sesión| } \\
\text {-Semana 27ä: Actividad } 15 \text { - Segunda Sesión } \\
\text {-Semana 28a: Actividad } 22\end{array}$ \\
\hline Мауо & $\begin{array}{l}\text {-Semana 29a: Actividad } 8 \\
\text {-Semana 30a: Actividad } 16 \\
\text {-Semana 31": Actividad } 23 \\
\text {-Semana 32a: Actividad } 24\end{array}$ \\
\hline
\end{tabular}




\section{ANEXO II. Registro de observación del alumnado}

\section{Registro de observación del alumnado}

Indicadores de observación

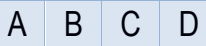

Respeta las normas básicas de comunicación: turno de palabra, mirar a los ojos al interlocutor.

Expresa sus opiniones y argumentos con calma.

Argumenta con claridad.

Escucha a los demás con atención.

Se dirige a docentes y a otros alumnos con respeto.

Manifiesta respeto por la igualdad entre todos.

Coopera sin discriminar.

Ayuda a los demás.

Mantiene buena relación con los compañeros.

Valora positivamente sus características personales.

Reconoce sus propias virtudes, habilidades y capacidades.

Identifica las emociones de los otros.

Respeta y valora las emocionesajenas.

Identifica y expresa sus emociones abiertamente

Valora positivamente sus emociones.

Identifica los méritos de otros.

Valora adecuadamente los méritos de los demás.

Celebra del éxito de los demás.

Identifica sus necesidades y establece metas adecuadas parasatisfacerlas.

Define sus metas y planifica para alcanzarlas.

Identifica situaciones en las que se da un locus de control interno.

Identifica situaciones en las que seda un locus de control externo.

Asigna el locus de control adecuado a sus propios actos y a las consecuencias de los mismos.

Identifica cuándo las consecuencias de sus actos y decisiones están verdaderamente bajo su control.

Define expectativas personales.

Asigna un valor proporcional a sus expectativas

Se motiva con el valor de sus expectativas. 


\section{ANEXO III. Cuestionario de autoevaluación docente}

\section{Cuestionario de autoevaluación docente}

\section{REFLEXIONO Y RESPONDO}

1. Procuro crear un ambiente de tranquilidad y confianza en el aula

2. Promuevo la participación de todos y la inclusión

3. Trato a todos mis alumnos con respeto y exijo que se traten de igual modo entre ellos

4. Planteo retos que se estén en su zona de desarrollo próximo, alcanzables y moderadamente difíciles.

5. Les estimulo a emprender actividades y les ayudo a que se fijen sus propias metas.

6. Les muestro la importancia de lo que están aprendiendo

7. Relaciono los contenidos con la realidad y sus intereses y experiencias.

8. Promuevo la empatía y el respeto a las diferencias

9. Planifico actividades colaborativas y cooperativas.

10. Procuro agrupamientos flexibles.

11. Les trasmito la idea de que son capaces y pueden lograr su objetivo.

12. Evalúo a mis alumnos respecto a sí mismos y no respecto a los compañeros.

13. Propongo metas divertidas y desafiantes que sean un reto personal para cada alumno.

14. Propongo actividades con dificultad creciente pero siempre con posibilidades de éxito.

15. Elijo refuerzos que sean alabanzas y celebraciones más que premios.

16. Evalúo sobre todo el esfuerzo más que el resultado.

17. No castigo el fracaso y celebro con entusiasmo sus triunfos

18. Les doy oportunidades de escoger temas, actividades, estrategias...

19. Procuro ofrecerles la información por diferentes cauces; visual, kinestésica, auditiva...

20. Varío los estímulos: preguntar, cambiar de actividad, lugar, agrupaciones, promover la participación.

21. Empleo recompensas y no castigos

22. Permito que expresen sus sentimientos y emociones, les escucho y muestro las propias.

23. Les muestro a menudo que son importantes.

24. Señalo con firmeza pero sin reproches las malas acciones.

25. Les ayudo a ponerse en lugar del otro; favoreciendo la empatía.

26. Estimulo el diálogo entre los alumnos y conmigo.

27. Establezco debates que promuevan sus opiniones y el juicio crítico.

28. Nombrolas emociones de forma incidental y en las actividades.

29. Procuro relatar experiencias y anécdotas como ejemplo de gestión de emociones.

30. Les enseño a darse instrucciones a sí mismos mientras actúan.

31. Les animo a repetir atribuciones adecuadas, primero en voz alta, luego en voz baja y, finamente, en silencio.

32. Les hago sentir que el resultado depende de ellos mismos y su trabajo.

33. No castigo los errores; alabo la persistencia en la tarea

SÍ NO OBSERVACIONES
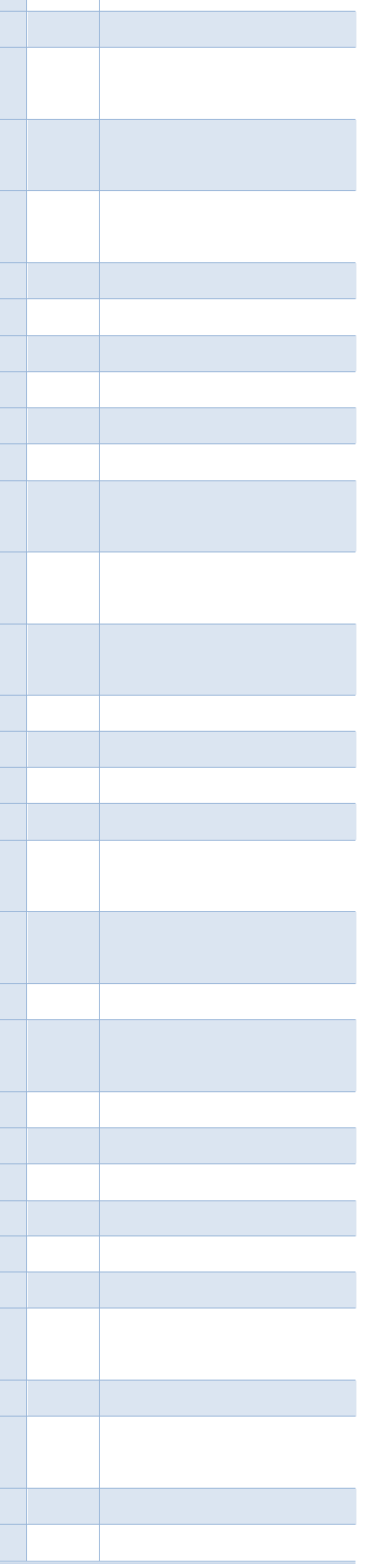